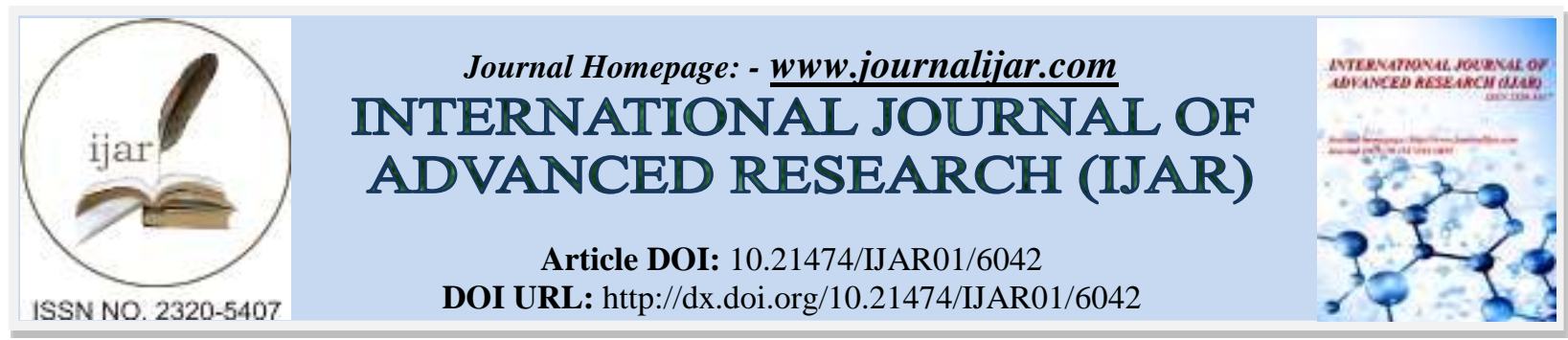

RESEARCH ARTICLE

\title{
ETUDE DE LA COMPOSITION CHIMIQUE DE L'HUILE ESSENTIELLE DE MONANTHOTAXIS CAPEA (ANNONACEAE), PLANTE ENDÉMIQUE D'AFRIQUE.
}

\section{Kouamé raphaël oussou ${ }^{1}$ jean brice boti ${ }^{2}$, coffi kanko ${ }^{2}$ and affouet muriel koffi ${ }^{2}$.}

1. Laboratoire de Chimie Organique et des substances Naturelles et; Université Jean Lorougnon Guédé Daloa BP 150 Daloa (Côte d'Ivoire).

2. Laboratoire de chimie Organique Biologique et Structurale, Université de Félix Houphouet Boigny Abidjan.

\section{Manuscript Info}

Manuscript History

Received: 12 October 2017

Final Accepted: 14 November 2017

Published: December 2017

Key words:-

Aromatic plants; essential oil;

Monanthotaxis capea.

\section{Abstract}

Monanthotaxis capea is an endemic plant of Côte d'Ivoire, it grows in the wild in the South of the country the study of the chemical composition of the essential oil of this plant showed that it contains more compounds of sesquiterpens which precocen I, neoiso menthyl acetat and sesquisabinen. This chemical composition is very different from that of literature found 13 years ago.

Copy Right, IJAR, 2017,. All rights reserved.

\section{Introduction:-}

Monanthotaxis capea fait partie de nombreuses espèces végétales d'Afrique intertropicales en voie d'extinction. Actuellement on ne connaît qu'une seule station de cette plante endémique de Côte d'Ivoire : la sous-préfecture d'Attobrou, la région d'Agboville.

Cette plante a cependant pu être préservée par certains habitants d'Attobrou, de Boguié et d'autres villages de la région qui en font la culture dans leurs vergers de café-cacao ou dans leur concession (Anoma, 1989; Aubreville, 1959)

En raison de leur parfum et de leurs vertus médicinales, les rameaux feuillés, tressés en couronne puis séchés font l'objet d'un commerce chez les populations d'Agboville. Cette activité commerciale était particulièrement importante avec le Ghana et le Nigeria. Dans ces deux pays, la plante était utilisée pour l'industrie cosmétique.

Trois travaux scientifiques ont été réalisés sur l'huile essentielle de cette espèce végétale:

YOBOUET, en 1996 a identifié un seul composé, le 4- phénylbut- 3-èn- 2- yl- acétate (E), de l'huile essentielle extraite des feuilles.

DOE en 1997 a mis en évidence 11 composés dont quatre majoritaires, le 4- phénylbut- 3-èn- 2- yle- acétate (E) (63\%), le 4-phénylbut-3-èn-2-ol(E) $(15,1 \%)$ et le 1-phénylbut-2-one $(6,5 \%)$, de l'essence des feuilles de cette plante. DOE a soumis cette huile à des tests de toxicité cutanée et oculaire sur des lapins. Ces tests ont révélé que cette huile essentielle est toxique pour les yeux mais ne l'est pas pour la peau. Elle pourrait donc être utilisée en application cutanée. 
Les travaux de MEVY réalisés en 2004 sur l'huile essentielle des feuilles de Monanthotaxis capea ont mis en évidence 28 composés avec aussi le 4- phénylbut- 3-èn- 2- yl- acétate (E) comme composé majoritaire (MEVY ; 2004)

En dehors de ces trois travaux aucun travail n'a été réalisé. Nous nous sommes proposé d'étudier 13 ans après MEVY, la composition chimique des huiles essentielles et d'en déterminer les grandes familles chimiques à savoir les composés hydrogénés et les composés oxygénés.

\section{Materiel Et Methodes:-}

Extraction des huiles essentielles:-

Les feuilles de Monanthotaxis capea proviennent de la région d'Atobrou, sous-préfecture (S/P) d'Agboville. Les huiles essentielles ont été obtenues par entrainement à la vapeur d'eau à l'aide d'un appareil de type Clévenger. Après extraction les huiles sont conservées à l'abri de la lumière et dans un congélateur $0^{\circ} \mathrm{C}$ avant leur analyse spectrale

\section{Analyse spectrale:-}

Pour l'analyse des extraits d'huiles essentielles nous avons utilisé les techniques d'analyse physicochimiques suivantes : la résonance magnétique nucléaire (RMN du proton et du carbone 13), la chromatographie en phase gazeuse (CPG) et le couplage CPG/SM. Cette analyse chimique a été réalisée en Corse (France) (Boti ,2007)

Les spectres de RMN sont enregistrés à l'aide d'un appareil de type Bruker (Bruker BioSpin AG). Le solvant d'élution est le chloroforme deutérié. Les déplacements chimiques ( $\delta$ en ppm) sont comparés au TMS pris comme référence interne.

Il est équipé d'une sonde de 5 à $10 \mathrm{~mm}$, opérant à 400,132 MHz pour le proton ou à 100,623 MHz pour le carbone 13 . Les enregistrements des spectres du carbone 13 ont été réalisés dans les conditions suivantes : Sonde de $5 \mathrm{~mm}$ : angle d'impulsion $45^{\circ}$; temps d'acquisition $=2,73 \mathrm{~s}$ correspondant à une acquisition de $64 \mathrm{~K}$ avec une largeur spectrale (SW) de $25000 \mathrm{~Hz}(250 \mathrm{ppm})$; résolution digitale de $0,183 \mathrm{~Hz} / \mathrm{pt} ; 70 \mathrm{mg}$ d'huile essentielle ont été dissouts dans $5 \mathrm{~mL}$ de $\mathrm{CDCl}_{3}$. Tandis que le nombre d'accumulations était compris entre 2000 et 5000 pour chaque enregistrement. Le découplage a été réalisé par champ pulsé «Composite Phase Decoupling ». Les données du signal de précession libre ont été multipliées avant la transformée de Fourrier par une fonction exponentielle (LB = 1,0 Hz).

\section{Caractéristiques physicochimique des huiles essentielles:-}

Cette étude a consisté en la détermination du rendement et en la mesure des grandeurs physico-chimiques telles que l'indice de réfraction, la densité et le $\mathrm{pH}$.

\section{Rendement :-}

Après 2 heures 30 min de distillation des organes végétaux, le rendement en huiles essentielles est calculé selon la formule suivante : $R(\%)=\frac{m_{2}}{m_{1}} .100$

Avec $\mathrm{R}=$ rendement en $(\%) ; \mathrm{m}_{1}=$ masse de l'organe végétal distillée $(\mathrm{g}) ; \mathrm{m}_{2}=$ masse de l'huile essentielle après 2 H 30 mn de distillation (g)

Détermination de l'indice de réfraction :-

Le réfractomètre utilisé est le réfractomètre d'ABBE à affichage numérique, il permet la détermination d'indices de réfraction compris entre 1,300 et 1,700. Le réfractomètre est relié à un thermostat qui permet de déterminer l'indice à $20^{\circ} \mathrm{C}$. Le principe de ce réfractomètre est basé sur la déviation angulaire provoquée par la réfraction de la lumière.

\section{Détermination de la densité:-}

La densité est déterminée avec un densitomètre à affichage numérique de type APPAR DMA 45. Elle a été réalisée aussi à $20^{\circ} \mathrm{C}$ comme précédemment. 
Détermination du potentiel hydrogène $(\mathbf{p H})$ :- Le $\mathrm{pH}$ des huiles essentielles est déterminé grâce à un $\mathrm{pH}$-mètre de type WPACD 7000 à affichage numérique.

\section{Resultats Et Discussion:-}

Tableau I:- Caractéristiques physicochimiques des huiles essentielles

\begin{tabular}{|c|c|c|c|c|}
\hline Rendement (\%) & Densité & $\mathrm{pH}$ & Indice de réfraction & Couleur \\
\hline 0,87 & 0,773 & 4,50 & 1,330 & Jaune pâle \\
\hline
\end{tabular}

Pour le rendement, nos résultats sont nettement supérieurs à ceux de GNASIMGBE et de MEVY qui ont trouvé respectivement 0,77\% et 0,70\% (Gnassingbé, 1997, Chalchat,1991), Les méthodes de distillation des organes végétaux pourraient expliquer cette différence de rendement. Les huiles essentielles de ces deux auteurs ont été obtenues par hydrodistillation, contrairement aux nôtres qui ont été extraites par entraînement à la vapeur d'eau à l'aide d'un distillateur de type Clevenger. La méthode d'extraction des huiles essentielles influencerait donc leur rendement.

Cette huile essentielle est acide ( $\mathrm{pH}=4,5)$.ce qui expliquerait son léger pouvoir antimicrobien (DOE, 1996). En effet, presque la quasi-totalité des microorganismes vivent à des $\mathrm{pH}$ neutres, (ou proches de 7). La présence des huiles essentielles dans le milieu de vie des microbes modifie le $\mathrm{pH}$ du milieu et donc agirait sur la croissance de ceux-ci.

Cette analyse a permis d'identifier 31 composés dont la teneur est supérieure ou égale à $0,1 \%$ dans l'huile des feuilles de Monanthotaxis capea. Ils sont présentés dans le tableau 2

Tableau 2 :- Composition chimique de l'huile essentielle des feuilles de $M$. capea

\begin{tabular}{|c|l|c|c|c|c|}
\hline $\mathbf{N}^{\circ}$ & Composés identifiés & IKA & IKP & Teneur \\
\hline 1 & $\alpha$ - pinène & 931 & 1022 & 0,2 \\
\hline 2 & $\beta$ - pinène & 970 & 1110 & 0,21 \\
\hline 3 & limonène & 1020 & 1199 & \\
\hline 4 & cis hydrate de sabinène & 1083 & 1541 & 0,16 \\
\hline $\mathbf{5}$ & citronellal & $\mathbf{1 1 3 1}$ & $\mathbf{1 4 7 9}$ & & $\mathbf{7 , 1 2}$ \\
\hline 6 & carvone & 1222 & 1739 & & 0,33 \\
\hline 7 & carvacryl méthyl oxide & 1232 & 1601 & \\
\hline 8 & géranial (citral) & 1237 & 1731 & & 0,1 \\
\hline 9 & menth-2-ène para 1,4 dihydroxy & 1245 & 2073 & & 0,11 \\
\hline $\mathbf{1 0}$ & néoiso acétate menthyl & $\mathbf{1 2 9 5}$ & $\mathbf{1 5 7 8}$ & & $\mathbf{1 7 , 0 1}$ \\
\hline 11 & géraniate de méthyle & 1301 & 1680 & & $\mathbf{4 , 9 6}$ \\
\hline 12 & cis acétate de carvyle & 1343 & 1767 & & 0,34 \\
\hline 13 & eugénol méthyle & 1367 & 2009 & & 1,9 \\
\hline 14 & $\beta$ - élemène & 1388 & 1589 & & 0,57 \\
\hline 15 & $\beta$ - funebrène & 1419 & 1607 & & 2,11 \\
\hline 16 & Trans $\alpha$ - bergamotène & 1432 & 1580 & & $\mathbf{4 , 3 8}$ \\
\hline 17 & trans cinnamate d'éthyle & 1434 & 2120 & & $\mathbf{4 , 4 4}$ \\
\hline 18 & thujopsène & 1435 & 1617 & & $\mathbf{6 , 8 3}$ \\
\hline $\mathbf{1 9}$ & sesquisabinène A & $\mathbf{1 4 3 7}$ & $\mathbf{1 6 3 6}$ & & $\mathbf{1 2 , 1 4}$ \\
\hline $\mathbf{2 0}$ & precocene I & $\mathbf{1 4 4 1}$ & $\mathbf{2 0 7 0}$ & & $\mathbf{3 0 , 1 2}$ \\
\hline 21 & (E) $\beta$ farnésène & 1448 & 1661 & & 0,11 \\
\hline 22 & $\alpha$ - humulène & 1456 & 1665 & & 1,95 \\
\hline 23 & $\beta$ - ionone & 1466 & 1936 & & 0,97 \\
\hline 24 & $\gamma$ - muurolène & 1471 & 1681 & & 0,12 \\
\hline 25 & $\beta$ - sélinène & 1483 & 1712 & & 0,7 \\
\hline 26 & bicyclo germacrène & 1494 & 1727 & & 0,4 \\
\hline 27 & Z $\alpha$ - bisabolène & 1516 & 1734 & & 0,21 \\
\hline 28 & $\delta$ - cadinène & 1752 & & 0,11 \\
\hline
\end{tabular}




\begin{tabular}{|l|l|r|r|r|}
\hline 29 & cèdrol & 1597 & 2121 & \multicolumn{1}{|c|}{0,32} \\
\hline 30 & patchoulol & 1657 & 2185 & 0,1 \\
\hline 31 & Acétate de 2E,6E farnésyle & 1822 & 2267 & 0,11 \\
\hline \multicolumn{1}{|c|}{ Total } & & $\mathbf{9 8 , 8 5}$ \\
\hline \multicolumn{2}{|c|}{ Composés oxygénés } & $\mathbf{4 5 , 1}$ \\
\hline Composés hydrocarbonés & 5,33 \\
\hline Monoterpènes hydrocarbonés & 8,34 \\
\hline Monoterpènes oxygénés & $\mathbf{4 8 , 4 2}$ \\
\hline Sesquiterpènes hydrocarbonés & $\mathbf{3 6 , 7 6}$ \\
\hline
\end{tabular}

IKA: Indice de Kovats sur colonne Apolaire ; IKP : Indice de Kovats sur colonne Polaire

La figure 1 présente les proportions des monoterpènes hydrocarbonés, monoterpènes oxygénés, sesquiterpènes hydrocarbonés et les sesquiterpènes oxygénés présents dans l'huile essentielle de Monanthotaxis capea

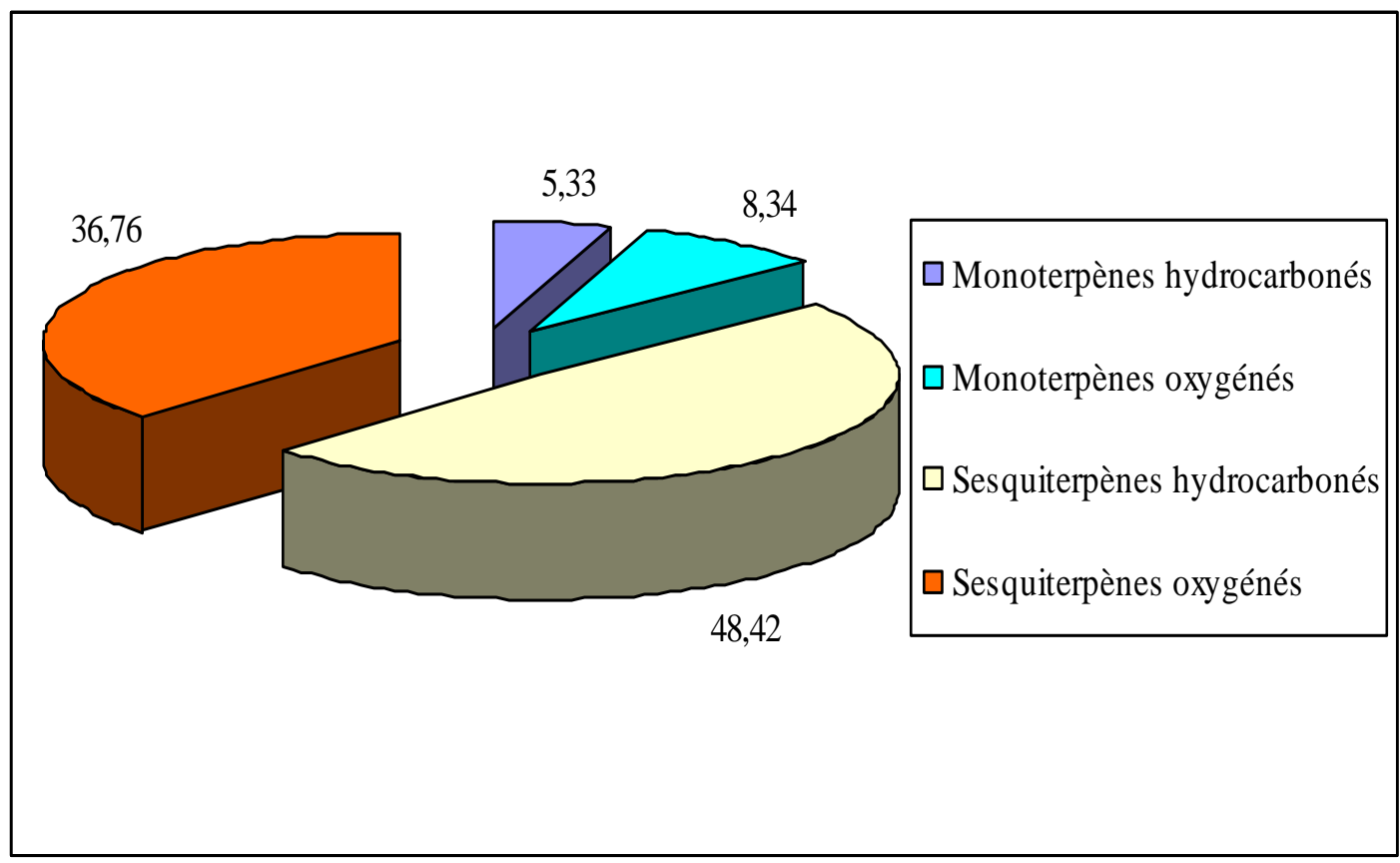

Figure 1 :- Proportion des composés présents dans l'huile essentielle de Monanthotaxis capea.

L'analyse de la composition chimique de l'essence de Monanthotaxis capea a permis d'identifier 31 composés qui représentent $98,85 \%$ de l'huile globale. Le tableau 2 donne les détails sur la composition chimique de cet échantillon. Parmi ces constituants, on distingue 11 monoterpènes : 6 composés oxygénés $(8,34 \%)$ et 5 hydrocarbures $(5,33 \%)$ ainsi que 20 sesquiterpènes : 10 composés oxygénés $(36,76 \%)$ et 10 hydrocarbures $(48,42 \%)$.

La fraction monoterpénique est caractérisée par le citronellal $(7,12 \%)$ et le trans $\alpha$ - bergamotène $(4,38 \%)$

La fraction sesquiterpénique est dominée par le précocène I (30,12\%), le néoiso acétate de menthyle (17,01\%), le sesquisabinène $\mathrm{A}(12,14 \%)$ et le thujopsène $(6,83 \%)$. Ces composés sont secondés par le géraniate de méthyle $(4,96 \%)$, le trans- cinnamate d'éthyle $(4,44 \%)$, le trans $\alpha$-bergamotène $(4,38 \%)$ et le $\beta$ - funebrène $(2,11 \%)$. Enfin viennent l' $\alpha$ - humulène $(1,95 \%)$ et le méthyle d'eugénol $(1,9 \%)$. Le tableau 3 permet de comparer la composition chimique des composés majoritaires de l'huile essentielle extraite des feuilles de $M$. capea de notre échantillon à celle de YOBOUET (Yobouet, 1996), DOE (1997) et de MEVY (2004) 
Tableau 3:- $\quad$ Composés majoritaires de Monanthotaxis capea de différents auteurs de Côte d'Ivoire (Yobouet, 1996 ; DOE ,1997 ; Mévy ,2004)

\begin{tabular}{|c|c|c|c|c|}
\hline Composés & $\begin{array}{c}\text { Notre } \\
\text { échantillon }\end{array}$ & $\begin{array}{c}\text { Doé } \\
(1997)\end{array}$ & $\begin{array}{c}\text { Yobouet } \\
(1996)\end{array}$ & $\begin{array}{l}\text { Mévy } \\
(2004)\end{array}$ \\
\hline Précocène I & 30,12 & & & \\
\hline Acétate de menthyle & 17,01 & & & \\
\hline Sesquisabinène A & 12,14 & & & \\
\hline Citronellal & 7,12 & & & \\
\hline Thujopsène & 6,83 & & & \\
\hline (E) Phényl-4 butène-3 ol-2 & & 15,10 & 80,0 & 18,30 \\
\hline (E) Acétate de méthyl-1 phényl-3 propèn-2 yle & & & & 68 \\
\hline Acétate de 4-phénylbut-3-ène-2-yle & & 63,0 & & \\
\hline
\end{tabular}

Nos résultats sont totalement différents de ceux de DOE, YOBOUET et de MEVY. En effet DOE a identifié l'acétate de 4-phénylbut-3-ène-2-yle comme composé majoritaire. MEVY a mis en évidence l'acétate de méthyl-1 phényl-3 propèn-2 yle. L'échantillon de YOBOUET est riche en phényl-4 butène-3 ol-2. La différence entre ces résultats témoigne de la complexité des composés de l'huile essentielle de Monanthotaxis capea. Les composés chimiques sont différents malgré le fait que la plante étudiée soit de la même espèce et récoltée dans la même localité (Agboville). Il se pourrait que les feuilles n'aient pas toutes été cueillies au même cycle végétatif de la plante et à la même période de l'année. Pour MEVY, les feuilles fraîches et les feuilles sèches n'ont pas la même composition chimique en huile essentielle. Par ailleurs la méthode d'extraction des huiles essentielles pourrait influencer la composition chimique. Nos échantillons d'huile ont été obtenus par entraînement à la vapeur d'eau, tandis que ceux des trois premiers auteurs (MEVY, YOBOUET et DOE) l'ont été par hydrodistillation. La figure2 donne la structure de quelques composés présents dans cette huile<smiles>COc1ccc2c(c1)OC(C)(C)C=C2</smiles>

Précocène I<smiles>CC(C)OC1C[C@H](C)CC[C@H]1C(C)C</smiles>

Acétate de menthyle

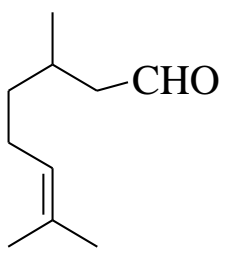

Citronellal<smiles>CC1=CC[C@]2(C)CCCC(C)(C)[C@@]2(C)[C@H]1C</smiles>

Cis thujopsène

Figure 2 :- $\quad$ Structure de quelques composés majoritaires de l'huile essentielle de Monanthotaxis capea.

\section{References Bibliograpiques:-}

1. Anoma G. et Ake Assi, Flore de la Côte d'ivoire : disparition de nombreuses espèces due à la destruction inconsidérée de l'espace naturel. Le cas de Monanthotaxis capea (E.G. et A. Camus) Verdc (Annonaceae) (1989). Bul. Soc. Bot. Fr, 136, Actual. Bot, pp.27-31

2. Aubreville A ; La flore forestière de la Côte d'Ivoire ( $2^{\text {ème }}$ édition, Tome premier Ed. C.T.F.T. Ngent sur Marne (1959), $360 \mathrm{p}$

3. Boti Jean Brice, Gérard Koukoua, Thomas Yao N'Guessan and Joseph Casanova; «Chemical variability of Conyza sumatrensis and Microglossa pyrifolia from Côte d'Ivoire». Flavour and Fragrance Journal (2007).; 22: 27-31.

4. Chalchat J. C., Garry R. Ph., Compositions chimiques de l'Huile Essentielle d'Angelica (Angelica archangelica)-Influence du séchage, Rivista Italiana EPPOS. 10, (1991), pp 468-478

5. Doé F. G. M., Monanthotaxis capea (E.G. et Camus) Verdc. Annonaceae : huile essentielle. Etude du capê des Agnis. Diplôme d'Etat de Docteur en pharmacie ; Université de Cocody-Abidjan (1997) ; 127 p

6. Gnassingbé B.; Etude de l'activité antibactérienne de cinq plantes de la flore ivoirienne : cas de Waltheria indica Linn. (Sterculiaceae) aspect botanique, phytochimique, pouvoir antimicrobien ; thèse de Pharmacie, Université de Cocody-Abidjan; (1997) 127 p.

7. Kanko C., Koukoua G., N'Guessan Y. T., Fournier J., Pradère J-P., Toupet L., Contribution à l'étude phytochimique de Lippia multiflora (Verbenaceae) C. R. Chimie 7

8. Mévy J.P., Bessière J.M., Pélissier Y., Masotti V., Ruzzier M., Rabier J., \& Viano J. Composition of the volatile constituents of the aerial parts of an endemic plant of ivory coast: Monanthotaxis capea (E.G. \& A. Camus) Verdc. Flavour and Fragrance Journal (2004), 19 : pp 526-528 
9. Oussou Kouamé Raphaël Séri Yolou Jean Brice Boti Kouadio Nathalie Guessennd Coffi Kanko Coffy Ahibo Joseph Casanova Etude Chimique et Activité Anti diarrhéique des Huiles Essentielles de Deux Plantes Aromatiques de la Pharmacopée Ivoirienne ; European Journal of Scientific Research (2008)ISSN Vol.24 No.1, pp. $94-103$

10. Tomi F., Nianga M., Casanova J., Identification of the Components of Xylopia aethiopica Essential Oil from Guinea Using carbon-13 NMR Spectroscopy, Journal of

11. Yobouet H. L. Contribution à l'étude botanique et analyses chimiques des huiles essentielles de 9 espèces végétales recensées en Côte d'Ivoire. Diplôme d'Etat de Docteur en pharmacie ; Université de Cocody-Abidjan (1996), $123 \mathrm{p}$. 